CNLS-94-01

\title{
Periodic Orbits and Shell Structure in Octupole Deformed Potentials
}

\author{
W.D. HEISS ${ }^{\star}$, R.G. NAZMITDINOV ${ }^{\star \star}$ 目 AND S. RADU ${ }^{\star}$ \\ * Centre for Nonlinear Studies and Department of Physics \\ University of the Witwatersrand, PO Wits 2050, Johannesburg, South Africa

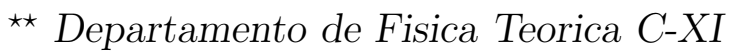 \\ Universidad Autonoma de Madrid, E-28049, Madrid, Spain
}

\begin{abstract}
The effect of an octupole term in a quadrupole deformed single particle potential is studied from the classical and quantum mechanical view point. Whereas the problem is nonintegrable, the quantum mechanical spectrum nevertheless shows some shell structure in the superdeformed prolate case for particular, yet fairly large octupole strengths; for spherical or oblate deformation the shell structure disappears. This result is associated with classical periodic orbits which are found by employing the removal of resonances method; this approximation method allows determination of the shape of the orbit and of the approximate octupole coupling strength for which it occurs. The validity of the method is confirmed by solving numerically the classical equations of motion. The quantum mechanical shell structure is analysed using the particle number dependence of the fluctuating part of the total energy. In accordance with the classical result this dependence turns out to be very similar for a superdeformed prolate potential plus octupole term and a hyperdeformed prolate potential without octupole term. In this way the shell structure is explained at least for some few hundred levels. The Fourier transform of the level density further corroborates these findings.
\end{abstract}

\footnotetext{
${ }^{1}$ on leave of absence from Joint Institute for Nuclear Research, Bogoliubov Laboratory of Theoretical Physics, 141980 Dubna, Russia
} 
PACS Nos.: 36.20.Kd, 36.40.+d, 21.10-k, 21.60.Cs, 05.45.+b 


\section{Introduction}

The shell structure is one of the most important quantum features of finite Fermi systems. The quantisation of a system of Fermions moving in a common potential leads to a bunching of levels in the single-particle spectrum, known as shells. When the levels of a bunch are filled the system is particularly stable; an additional particle fills a level in the next bunch at considerably higher energy and therefore produces a less stable system. In atoms and nuclei the shell effects are discerned by the deviations of the binding energies from the smooth variation obtained from the liquid-drop model or as oscillations in the radial density distribution as a function of particle number and of deformation [1]. As it was pointed out in [2, 3] the shells are a global phenomena not only for spherical systems. When a spherical shell is only partially filled, the higher degeneracy in the single-particle spectrum of a deformed mean field can lead to a breaking of spherical symmetry, and it therefore gives rise to a deformed equilibrium shape.

A deep understanding of shell structure phenomena in terms of classical trajectories has been achieved by Balian and Bloch [⿴囗 히 based on the periodic orbit theory of Gutzwiller [5]. According to the semiclassical theory [4] the frequencies in the level density oscillations of single-particle spectra of nuclei are determined by the corresponding periods of classical closed orbits. The short periodic orbits give the major contribution to the gross shell structure [1, 6, 7, 8]. Depending on the particular mean field potential a deviation from spherical symmetry can lead to chaotic motion in the corresponding classical problem, and the shell structure of the corresponding quantum spectrum is affected or even destroyed depending on the degree of chaos [9, 10, 11].

Atomic clusters are another area which can serve as testing ground for these ideas. Recent experimental results on metallic clusters reporting abundance variations in mass spectra, ionisation potentials, static polarisabilities and collective giant dipole resonances, barrier shapes and fragmentation provide us with striking manifestations of shell structure effects related to a quantised motion of the valence electrons [12, 13]. The correspondence of the electronic shell structure in spherical clusters to the closing of major quantal shells [14, 15, 16] caused considerable interest in using nuclear shell model type calculations for the description of metallic clusters [17, 18, 19, 20]. It turns out that phenomenological potentials used traditionally in nuclear physics serve a purpose similar to those obtained within the KohnSham density-functional method [21] if the relevant parameters are adjusted appropriately. 
Typical potentials are the Woods-Saxon (and its various modifications) and the modified Nilsson potential without spin-orbit term. The considerable lowering of the computational time due to their simple analytical form renders an analysis of the stability of large metallic clusters feasible. Naturally, the shell numbers have to be larger than the ones used in nuclear physics in accordance with the larger number of valence electrons considered for metallic clusters. Recently, the semiclassical analysis has been successfully applied to explain shell structure produced by valence electrons [22, 23, 24] in different metals. As it was mentioned before, obviously, shell structure in the quantum mechanical spectrum is associated with periodic orbits in the corresponding classical problem. Moreover, following work by Balian and Bloch [4], Nishioka, Hansen and Mottelson [22] predicted the supershell, which is a beating pattern, when the regular oscillations reflecting the main shells are enveloped by an oscillating amplitude of lower frequency. As was argued in the case of a spherical cavity [4], the beating pattern is caused by the interference between the two most important classical trajectories which are characterised by orbits of a triangular and square shape. For mesoscopic objects like clusters, deformations, i.e. deviations from spherical symmetry of the potential, are as important as in the nuclear physics context (for example, the smaller peaks in the cluster abundances correspond to unfilled shells or deformed shapes of clusters [25]). Direct information about the deformation of clusters can be obtained from an analysis of a splitting of the plasmon resonance or from a more detailed investigation of mass abundance.

Based on a simple analysis of the geometry of closed classical orbits, Mottelson [26, 1] predicted a tendency for a large system (heavy nuclei) with a 2:1 quadrupole deformation to exploit the octupole degree of freedom. For nuclei this idea was supported by Nilsson+Strutinsky shell-structure energy calculations [27], by a systematic study of octupole correlations within a microscopic approach [28, 29] and by a qualitative analysis of the dynamical symmetry of the anisotropic harmonic oscillator [30]. Hamamoto et al [31] investigated the importance of nonaxial octupole deformation for many-body system within a harmonic oscillator model. More recently, authors in [18 used a deformed Woods-Saxon potential, including quadrupole, octupole and hexadecupole terms, to calculate the groundstate deformation of sodium clusters.

Inclusion of an octupole term in addition to a quadrupole term renders the classical single particle motion nonintegrable. In fact, the system turns out to be chaotic. In a recent investigation [32] the study of classical motion in a cavity with oscillating walls of even and odd higher order multipoles has led to interesting conclusions about elastic versus dissipative 
behaviour of a noninteracting gas depending on the integrability or nonintegrability of the equations of motion.

In the present paper we thoroughly investigate a simplified model. We leave out terms, which, albeit physically important, are prone to blur the analysis when the interest is focussed on the essentials, in particular a distinction between orderly and chaotic motion in many body systems, like nuclei and metallic clusters. Since our interest is directed not only towards the classical but also the corresponding quantum mechanical motion, we leave out the spin-orbit term and the $(\vec{l})^{2}$-term present in the Nilsson model so as to render as closely as possible the analogy between the classical and quantum cases. An analysis of classical trajectories in a quadrupole deformed harmonic oscillator has shown that the spin orbit term leads to chaotic motion [33]. Likewise, the $(\vec{l})^{2}$-term gives rise to chaotic behaviour [34] even without the octupole term. Despite and because of the simplifications, such a model allows an understanding of the main features of shell structure effects in super (hyper) deformed nuclei [1], 30] and in deformed clusters (see, for example, [12, 35] and references quoted therein). Recent experimental data of superdeformed K-isomers in nuclei [36] and electronic shell structure effects in metallic clusters [12] seem to indicate the manifestation of oblate deformation in nuclei and small clusters. In the present paper we investigate the effect of the octupole term for prolate and oblate deformation; the latter case has not been dealt with in [32].

One major result of the present paper is a classical analysis which demonstrates that the prolate case including octupole deformation is still quasi-integrable. In this way the quantum mechanical shell structure found earlier [11] is given a proper theoretical foundation. One may speculate that this is the reason why for large quadrupole deformation prolate-octupole nuclei(clusters) are more stable than the oblate-octupole ones. For metallic clusters finite temperature should be considered [15, 16]; however, our interest is focussed on shell structure whose character is unaffected by temperature except for the amplitude. The detailed analysis of magic numbers, their changes under variation of the octupole strength and the possibility of supershell structure induced by the octupole term is a further major contribution of this paper. 


\section{The model}

We investigate the classical and quantum mechanical motion in the potential

$$
V(\varrho, z)=\frac{m}{2} \omega^{2}\left(\varrho^{2}+\frac{z^{2}}{b^{2}}+\lambda \frac{2 z^{3}-3 z \varrho^{2}}{\sqrt{\varrho^{2}+z^{2}}}\right) .
$$

For $b>1(b<1)$ this is a quadrupole deformed harmonic oscillator of prolate (oblate) shape with an additional octupole term; in fact the term proportional to $\lambda$ is $r^{2} P_{3}(\cos \theta)$ with $P_{3}$ the third order Legendre polynomial. We use cylindrical coordinates $z$ and $\varrho=\sqrt{x^{2}+y^{2}}$. For $\lambda \neq 0$ this is a two degrees of freedom system which is non-integrable.

The exact classical orbits are obtained numerically by integrating the equations of motion, viz.

$$
\begin{aligned}
\ddot{\varrho} & =-\frac{\partial}{\partial \varrho} V(\varrho, z) \\
\ddot{z} & =-\frac{\partial}{\partial z} V(\varrho, z) .
\end{aligned}
$$

It is convenient to use the energy as one initial condition; because the potential obeys the scaling law $V(\gamma \varrho, \gamma z)=\gamma^{2} V(\varrho, z)$ one value of the energy yields orbits of all energies. The equation for $\varrho$ would contain the term $p_{\phi}^{2} / \varrho^{3}$ on the right hand side of Eq.(2) if a finite value of the (conserved) angular momentum $p_{\phi}$ would be considered. However, as discussed in the following section, $p_{\phi}=0$ is sufficient for all classical aspects relevant in this paper.

Our choice of the octupole term ensures that we have a genuine bound state problem for $\lambda<\lambda_{\text {crit }}$. Here $\lambda_{\text {crit }}$ is defined to be the value for which the potential no longer binds, and for $|\lambda|>\lambda_{\text {crit }}$ the potential tends to $-\infty$ along one or two directions in the $\varrho$ - $z$-plane. The direction and the value of $\lambda_{\text {crit }}$ depend on the quadrupole deformation $b$. For prolate nuclei $(b>1), \lambda_{\text {crit }}=1 /\left(2 b^{2}\right)$ and the potential opens its valley along the positive (negative) $z$-direction for negative (positive) $\lambda$. For oblate nuclei $(b<1)$ the other possible direction along the line $\varrho=\operatorname{sign}(\lambda) \beta z$ with $\beta \approx 0.4$, is of increasing importance. At the value $b \approx 0.58$ valleys along the two directions $\varrho=0$ and $\varrho=0.4 z$ open simultaneously for $\lambda_{\text {crit }} \approx 1.5$ while for a still smaller value of $b$, say for $b=0.5$, the valley along the direction $\varrho=0.4 z$ opens for $\lambda_{\text {crit }} \approx 1.64$ while the one along the $z$-axis now opens for a larger value of $\lambda$. Analytic expressions for $\lambda_{\text {crit }}$ and the direction $\beta$ as functions of $b$ are given in Appendix A. 


\section{Classical perturbative treatment}

To understand the occurrence of shell structure in the corresponding quantum mechanical problem we present a classical analysis in which it becomes obvious that, for the prolate case, the problem is approximately equivalent to an integrable problem even for finite values of $\lambda$. In fact it is shown in what follows that, within the approximation used, the motion in the $z$ and $\varrho$-direction becomes uncoupled whereby the motion in the $z$-direction depends on $\lambda$. In this way frequencies in either direction can be clearly defined with the effect that the corresponding winding number, i.e. the ratio $\omega_{\varrho} / \omega_{z}$, becomes a simple function of $\lambda$. This provides the basic mechanism for the occurrence of shell structures for the quantum mechanical problem; in fact it provides classical evidence for the quantum mechanical finding that the spectrum for specific values of $\lambda$ is nearly equivalent to a spectrum with $\lambda=0$ but with a larger value of the quadrupole parameter $b$. We stress that the agreement between the perturbative results and the exact orbits is excellent.

The method employed is based on the 'removal of resonances' approach which amounts to a particular averaging procedure used in secular perturbation theory [37]. The complete Hamilton function written in terms of the angle and action variables of the unperturbed problem (see Appendix B) reads

$$
H\left(J_{\varrho}, J_{z}, \alpha_{\varrho}, \alpha_{z}\right)=\omega\left(J_{\varrho}+\frac{1}{b} J_{z}+\lambda \frac{\sqrt{b J_{z}} \sin \alpha_{z}\left(2 b J_{z} \sin ^{2} \alpha_{z}-3 J_{\varrho} \sin ^{2} \alpha_{\varrho}\right)}{\sqrt{b J_{z} \sin ^{2} \alpha_{z}+J_{\varrho} \sin ^{2} \alpha_{\varrho}}}\right) .
$$

The frequencies $\omega_{z}=\frac{\partial H}{\partial J_{z}}$ and $\omega_{\varrho}=\frac{\partial H}{\partial J_{\varrho}}$ are given by

$$
\begin{aligned}
\omega_{z}(\vec{J}, \vec{\alpha}) & =\frac{\omega}{b}\left(1+\lambda \frac{b \sin \alpha_{z}\left(4 b^{2} J_{z}^{2} \sin ^{4} \alpha_{z}+6 b J_{z} J_{\varrho} \sin ^{2} \alpha_{\varrho} \sin ^{2} \alpha_{z}-3 J_{\varrho}^{2} \sin 4 \alpha_{\varrho}\right)}{2 \sqrt{b J_{z}}\left(b J_{z} \sin ^{2} \alpha_{z}+J_{\varrho} \sin ^{2} \alpha_{\varrho}\right)^{\frac{3}{2}}}\right) \\
\omega_{\varrho}(\vec{J}, \vec{\alpha}) & =\omega\left(1-\lambda \frac{\sin \alpha_{z} \sin ^{2} \alpha_{\varrho} \sqrt{b J_{z}}\left(8 b J_{z} \sin ^{2} \alpha_{z}+3 J_{\varrho} \sin ^{2} \alpha_{\varrho}\right)}{2\left(b J_{z} \sin ^{2} \alpha_{z}+J_{\varrho} \sin ^{2} \alpha_{\varrho}\right)^{\frac{3}{2}}}\right) .
\end{aligned}
$$

It is clear from these expressions that, for small values of $\lambda$, the ratio $\omega_{\varrho} / \omega_{z}$ is essentially determined by $b$. For prolate superdeformation, when $b=2$ or larger, one can expect that averaging over the fast moving angle $\alpha_{\varrho}$ of the Hamilton function should yield a good approximation. Since the Hamilton function is periodic in both angles, this approximation amounts to keeping the zero order term of its Fourier expansion in the fast moving angle [37. Since integration of the Hamilton function over $\alpha_{\varrho}$ can be done analytically, this approximation appears particularly attractive. 
After averaging and then rewriting the action variables and the remaining angle $\alpha_{z}$ in terms of the original momentum and coordinate values we obtain from Eq.(3)

$$
H_{\mathrm{av}}=\frac{p_{\varrho}^{2}+p_{z}^{2}}{2 m}+\frac{m \omega^{2}}{2}\left[\varrho^{2}+\frac{z^{2}}{b^{2}}+\lambda \xi^{2} \frac{\operatorname{sign}(z)}{2 \pi}\left(8 \frac{z^{2}}{\xi^{2}} K\left(-\frac{\xi^{2}}{z^{2}}\right)-3 \pi F_{21}\left(\frac{1}{2}, \frac{3}{2}, 2 ;-\frac{\xi^{2}}{z^{2}}\right)\right)\right]
$$

where $\xi^{2}=2 J_{\varrho}^{(0)} /(m \omega)=\varrho^{2}+p_{\varrho}^{2} /(m \omega)^{2}$ which is a constant within the approximation. Here $F_{21}$ and $K$ are the hypergeometric function and the first elliptic integral, respectively.

With Eq.(5) we have effectively reduced the full problem to an integrable problem as the motion in the two coordinates is uncoupled. The motion in the $z$-coordinate is determined by an effective potential which now depends also on the action $J_{\varrho}$ in addition to its dependence on $\lambda$. Note that the effective potential originating from the octupole term depends only on $z / \xi, \xi \sim \sqrt{J_{\varrho}}$; this means that, depending on the action residing in the $\varrho$-motion, the amplitudes of the vibrations in the $z$-motion are increased or decreased. The potential of the $z$-motion is no longer harmonic. Because of its dependence on $\lambda$ and $J_{\varrho}$ we now expect ratios $\omega_{\varrho} / \omega_{z}$ which are different from the unperturbed value $b$ and which depend on $\lambda$ and $J_{\varrho}$. Also note that the large $z$ behaviour coincides with that of the complete problem which means that $\lambda_{\text {crit }}=1 /\left(2 b^{2}\right)$ still holds. In Fig.(1) we illustrate the effective potential.

A few comments are in order with regard to non-zero angular momentum. The additional term $p_{\phi}^{2} / \varrho^{2}$ in the Hamilton function does not alter our procedure nor the results based upon it. In fact, the $\varrho$-motion remains unperturbed including the centrifugal term, and for the harmonic oscillator the frequency $\omega_{\varrho}$ remains therefore equal to its zero angular momentum value. This is significant in the quantum mechanical context as the ratios $\omega_{\varrho} / \omega_{z}$ are independent of the angular momentum just like in an unperturbed harmonic oscillator. It is by this mechanism that we can explain pronounced shell structure for $\lambda \neq 0$, i.e. shells containing all values of angular momentum which are allowed quantum mechanically. We return to this point in the subsequent section.

The approximation procedure seems to be useful only for the super- and hyperdeformed prolate case. In the superdeformed oblate case $(b=1 / 2)$ one would, at first glance, consider averaging over $\alpha_{z}$. However, the zeroth order term vanishes now as the octupole term is an odd function of $\sin \alpha_{z}$. The first order term in the Fourier series no longer decouples the coordinates $\varrho$ and $z$ so that no gain is made. For the spherical case $(b=1)$ a canonical transformation could in principle enforce one of the (transformed) frequencies to be faster than the other. However, this procedure again is not useful since the slow frequency turns out to be a complicated function of time, in other words, the ratios $\omega_{\varrho} / \omega_{z}$ fail to be con- 
stant rational numbers. In simple terms, this discussion shows that only the fairly strongly deformed prolate case remains close to integrability when the octupole term is switched on. This is the classical explanation for the existence of shell structure in the quantum spectrum which occurs only for the prolate case [11]. As we see below the spherical and the oblate case soon become chaotic classically when the octupole is switched on, which prevents occurrence of shell structure in the corresponding quantum spectrum.

The frequency $\omega_{z}$ as determined by the approximation implied by Eq.(5) is given by $\omega_{z}=2 \pi / T$ where

$$
T=\sqrt{2 m} \int_{z_{\min }}^{z_{\max }} \frac{\mathrm{d} z}{\sqrt{E_{z}-U(z)}}
$$

with $E_{z}=E-E_{\varrho}=E-m \omega^{2} \xi^{2} / 2$ and $U(z)$ is taken from Eq.(5) and reads

$$
U(z)=\frac{m \omega^{2}}{2}\left[\frac{z^{2}}{b^{2}}+\lambda \xi^{2} \frac{\operatorname{sign}(z)}{2 \pi}\left(8 \frac{z^{2}}{\xi^{2}} K\left(-\frac{\xi^{2}}{z^{2}}\right)-3 \pi F_{21}\left(\frac{1}{2}, \frac{3}{2}, 2 ;-\frac{\xi^{2}}{z^{2}}\right)\right)\right] .
$$

The period $T$ can be evaluated numerically. In Fig.(2) we display the ratio $\omega_{\varrho} / \omega_{z}$ as a function of $\lambda$ for a few parameter values $\xi$. Starting at its unperturbed value $b=2$ for $\lambda=0$, it turns out that the ratio of the frequencies is a monotonously increasing function of $\lambda$ for $0 \leq \lambda<\lambda_{\text {crit }}$ for a large range of $\xi$-values, for which it is virtually independent of $\xi$; only when $\xi^{2}$ approaches its upper limit $2 E /\left(m \omega^{2}\right)$ can a notable $\xi$-dependence be discerned. We note that the integral in Eq.(6) can be evaluated analytically for $\xi=0$; in this case the ratio reads

$$
\frac{\omega_{\varrho}}{\omega_{z}}=\frac{b}{2}\left(\frac{1}{\sqrt{1+\lambda / \lambda_{\text {crit }}}}+\frac{1}{\sqrt{1-\lambda / \lambda_{\text {crit }}}}\right)
$$

which is the top curve drawn in Fig.(2). Simple, i.e. short periodic orbits occur when the frequency ratio becomes a rational number $p / q$ with small values of $p$ and $q$. From Fig.(2) we read off that around $\lambda=0.66 \lambda_{\text {crit }}$ the ratio is $5: 2$ and for $\lambda=0.8 \lambda_{\text {crit }}$ it is $3: 1$. In Figs.(3) we display the most important periodic orbits. If the approximation is meaningful this finding implies that for these particular values of $\lambda$ all orbits (except the ones with $E_{z} \gtrsim 0$ ) should be periodic with the respective winding numbers. The consequence for the quantum spectrum is clear: the parameters $b=2$ and $\lambda=0.66 \lambda_{\text {crit }}$ should yield a spectrum similar to that for the parameters $b=5 / 2$ and $\lambda=0$; likewise, $b=2$ and $\lambda=0.8 \lambda_{\text {crit }}$ should be nearly equivalent to $b=3$ and $\lambda=0$. In the following section we see a convincing confirmation of this classical prediction of quantum shell structure. 
One expects a similar pattern when starting from the outset, say, with $b=5 / 2$, since the onset of classical chaos seems to be delayed the larger the value of $b$. In fact, one finds appropriate $\lambda$-values for which the winding numbers become $3: 1$ (for $\lambda=0.63 \lambda_{\text {crit }}$ ) and 7:2 (for $\lambda=0.76$ ). Starting with $b=3$ one finds $7: 2$ and even 4:1, i.e. we have the the "chain of shells" $2: 1 \rightarrow 5: 2 \rightarrow 3: 1 \rightarrow 7: 2 \rightarrow 4: 1 \ldots$ We mention here the $7: 2$ orbit to demonstrate the principle, but we have left out the 7:3 in the previous paragraph although it is there between the $2: 1$ and 5:2. As a matter of fact we have left out all ratios $p / q$ with $p>7$ because they do not feature as prominently in the quantum spectrum as the shortest orbits with $p<7$. But we can draw the conclusion that an octupole term in a prolate deformed oscillator produces essentially a pure but more deformed prolate oscillator. This explains the occurrence of shell structure in the corresponding quantum spectrum at the particular $\lambda$-values for which the effective value of $b$ assumes a rational number with small value of the numerator and denominator.

We have tested the validity of the approximation procedure by looking at a large variety of exact orbits obtained from solving Eq.(2) numerically. In Fig.(4a) surfaces of section are displayed for $\lambda=0.63 \lambda_{\text {crit }}$ for a number of initial conditions. The slight discrepancy between the values 0.66 found from Fig.(2) and 0.63 used in Fig.(4a) reflects the difference between the approximation and the exact situation. Like in Fig.(2) the actual ratio when determined numerically is an increasing function of $\lambda$. It turns out that the actual ratio is slightly larger than 5:2 for $\lambda=0.66 \lambda_{\text {crit }}$ and is closest to $5: 2$ for $\lambda=0.63 \lambda_{\text {crit }}$. What meets the eye when looking at Fig.(4a) are the large stability islands around the 5:2 orbit. Note that at the periphery of the surface which is chosen to be the $\varrho=0$ plane we have $\xi^{2}=0$ while $\xi^{2}$ attains its maximum value towards the centre. In this way we sample the whole range. Of course we do not obtain an exact 5:2 orbit for a large range of $\xi$-values as the approximation would suggest; we do get the one in the centre of the islands. However, while most orbits are quasiperiodic and produce tori, their winding numbers are all very close to $5: 2$, for instance 70:27 or 70:29. This applies to most of phase space, deviations are noticeable only towards the centre of the section. In accordance with Fig.(2) there we get orbits with smaller winding numbers such as 7:3 and even 3:2. However, more than $85 \%$ of phase space is dominated by orbits with winding numbers very close to 5:2. The analogy of the parameter set $b=2, \lambda=0.63 \lambda_{\text {crit }}$ with $b=5 / 2, \lambda \approx 0$ goes even further in that not only the shape of the trajectory of the actual 5:2 orbit is the same but also that of the unstable orbit associated with the intersection points of the separatrix (a separatrix similar to the one displayed in 
Fig.(4a) is produced by the parameter set $b=5 / 2, \lambda=0.05 \lambda_{\text {crit }}$ ). Mutatis mutandis similar statements hold for the comparison of the parameter set $b=2, \lambda / \lambda_{\text {crit }}=0.76$ (see Fig.(4b)) and $b=3, \lambda \approx 0$. Note the small region where the onset of chaotic motion can be discerned; by and large the motion still appears close to integrabilty although $\lambda$ is fairly close to $\lambda_{\text {crit }}$.

For comparison we display surfaces of sections in Fig.(5) for the spherical and oblate case. Even though the diagrams represent results for smaller values of $\lambda / \lambda_{\text {crit }}$ the overall onset of chaos is obvious. Here we note that for the special value $b \approx 0.58$ discussed in section 2 the onset of chaos occurs for an even smaller value of the octupole strength, i.e. $\lambda / \lambda_{\text {crit }} \gtrsim 0.25$.

\section{Quantum mechanical results}

The quantum mechanical treatment is straight-forward in principle. In the spirit of previous work [38] we use for the full problem which is of the form $H_{0}+\lambda H_{1}$ a representation with $H_{0}$ diagonal. The basis chosen is referred [1] to as the basis using the asymptotic quantum numbers $n_{\perp}, n_{z}$ and $\Lambda$ where $n_{\perp}=n_{+}+n_{-}$and $\Lambda=n_{+}-n_{-}$. Here the quantum numbers $n_{+}$and $n_{-}$are the eigenvalues of $\left(A_{+}\right)^{\dagger} A_{+}$and $\left(A_{-}\right)^{\dagger} A_{-}$, respectively, where, in terms of the usual boson operators $a_{x}$ and $a_{y}$, we use $A_{ \pm}=\left(a_{x} \mp i a_{y}\right) / \sqrt{2}$. For a fixed value of $\Lambda$ (which is the quantised analogue of the classical $p_{\phi}$ ) this leaves two quantum numbers (reflecting the two degrees of freedom) to enumerate the rows and columns of the matrix problem. For $\Lambda=0$ the diagonal entries of $H_{0}$ are thus $\varepsilon_{n_{\perp}, n_{z}}^{0}=\hbar \omega\left(n_{\perp}+1+\left(n_{z}+1 / 2\right) / b\right)$. The matrix elements of $H_{1}$ are obtained from those of $z \sim\left(a_{z}^{\dagger}+a_{z}\right)$ and $\varrho^{2} \sim\left(A_{+}\left(A_{+}\right)^{\dagger}+A_{-}\left(A_{-}\right)^{\dagger}+\right.$ $\left.A_{+} A_{-}+\left(A_{-}\right)^{\dagger}\left(A_{+}\right)^{\dagger}\right)$. To get the matrix elements of $1 / \sqrt{\varrho^{2}+z^{2}}$ in a numerically consistent way, we first calculate the matrix elements $S_{m, n}$ of $\varrho^{2}+z^{2}$ from which the inverse square root is obtained using $S^{-1 / 2}=U \cdot D^{-1 / 2} \cdot U^{\dagger}$ where $D=U^{\dagger} \cdot S \cdot U$ is the diagonal form of the positive definite matrix $S$ and $U$ is the orthogonal matrix which diagonalises $S$. To ensure also numerically that $S$ has only positive eigenvalues it is important that the matrix for $z^{2}$ is obtained by squaring $z$ and not by evaluating analytically the matrix elements from $\left(a_{z}^{\dagger}+a_{z}\right)^{2}$; inconsistencies are otherwise introduced due to truncation. In this way, we also ensure that the truncated matrices $S^{-1 / 2}$ and the representation of $2 z^{3}-3 z \varrho^{2}$ commute and that their product is a symmetric matrix. The effect of truncation was tested by looking at the variation of the lower end of the spectrum when the dimension of the matrices was increased. There is certainly a dependence on $b$ and $\lambda$. For $\lambda \leq 0.9 \lambda_{\text {crit }}$ and $0.5 \leq b \leq 2$ the variation was less than $1 \%$ for the first 300 levels obtained from $1600 \times 1600$ dimensional 
matrices.

In Fig.(6a) we illustrate the spectrum so obtained as a function of $\lambda$ for $b=2$. The shell structure at about $\lambda=0.63 \lambda_{\text {crit }}$ and $\lambda=0.76 \lambda_{\text {crit }}$ is clearly discernible. While Fig.(6a) presents the spectrum for $\Lambda=0$ we illustrate in Fig.(6b) the whole spectrum which is a superposition of all possible $\Lambda$-values. Here the shell structure is even more pronounced which is expected since, according to the discussion in section 3, the orbits will have the same winding numbers independent of the angular momentum; as a consequence, the quantum spectra will have shell structures similar to an unperturbed oscillator. This is a prototype case for employing Strutinsky's procedure [2, 3] to exhibit the magic numbers associated with the shells. However, while we follow the basic idea of Strutinsky's method, we use a somewhat different approach which appears more suitable in our case; this is presently discussed.

It is clear that for the plain quadrupole deformed oscillator, supershell structure in the sense of [22] cannot occur [39] since there is basically only one orbit for each coordinate; it is the sinusoidal motion characterised by the respective frequency associated with that coordinate. The consequences for the quantum spectrum are well known [四]: one obtains the Fibonacci numbers as degeneracies in the equidistant spectrum. If the frequencies ratios are simple rationals, there are systematic repetitions of the same degeneracies [40, 30]. This is in contrast to, say, the motion in a cavity or in a Woods-Saxon potential where basically different classical orbits such as the triangle, the square, the pentagon and so forth exist thus giving rise to interference of different shells in the quantum spectrum. One of the questions addressed in this paper is the possible occurrence of supershell effects when a quadrupole deformed harmonic oscillator is perturbed by an anharmonic term, i.e. the octupole deformation.

To analyse the quantum spectrum we proceed in the orthodox fashion in that the total energy $E_{\text {tot }}(N, \lambda)=\sum_{i}^{N} \varepsilon_{i}(\lambda)$ is approximated by a smooth average function $S(z, \lambda)$ and the fluctuating difference

$$
\delta E(N, \lambda)=E_{\text {tot }}(N, \lambda)-S(N, \lambda)
$$

is then subjected to further investigation. The finding of a suitable form for the average function $S(z, \lambda)$ is facilitated in our case as it is well known [1] that the leading term of $E_{\text {tot }}(N, \lambda)$, as a function of $N$, is proportional to $N^{4 / 3}$. We determine the five constants $a_{0}(\lambda), \ldots, a_{4}(\lambda)$ in $S(z, \lambda)=\sum_{k=0}^{4} a_{k}(\lambda) z^{k / 3}$ by a least square fit which turns out to be 
perfectly satisfactory for all values of $0 \leq \lambda<\lambda_{\text {crit }}$.

In Fig.(7) the fluctuating part $\delta E(N, \lambda)$ is presented as a contour plot. The diagram refers to $b=2$ and displays the ranges $100 \leq N \leq 700$ and $0 \leq \lambda<\lambda_{\text {crit }}$. For $\lambda=0$ (the bottom horizontal line) we clearly discern the shell structure of the plain deformed oscillator. Note that the sharp minima (dark shadowing) occur at the positions $N$ where a shell is closed; the distances are proportional to $N^{3}$. When $\lambda$ is switched on the shell structure persists to a great extent; only when $\lambda$ approaches its critical value (top horizontal line) the structure begins to be washed out. There are local minima discernible at $\lambda / \lambda_{\text {crit }} \approx 0.76$. This is a reflection of the enhanced shell structure discussed in the previous section. Below we pursue this point in more detail. The finer structure and in particular the detailed shell structure is obtained from the second derivative of $\delta E(N, \lambda)$ to which we turn next.

In Figs. (8) we have plotted $g(E)=\delta E(N+1, \lambda)+\delta E(N-1, \lambda)-2 \delta E(N, \lambda)$ as a function of $N^{1 / 3}$ for a few characteristic values of $\lambda$. The peaks of the plots represent the magic numbers which characterise the shells, and the heights of the peaks reflect the energy distance from one shell to the next. In Fig.(8a) the essentially unperturbed result $\left(\lambda / \lambda_{\text {crit }}=0.15\right)$ is presented for demonstration. The second row displays the results for the particular values of $\lambda$ for which the winding numbers are 5:2 $\left(\lambda / \lambda_{\text {crit }}=0.63\right)$ and 3:1 $\left(\lambda / \lambda_{\text {crit }}=0.76\right)$, respectively. The magic numbers and the heights of the peaks agree well with those which are obtained from the unperturbed $(\lambda=0)$ quadrupole deformed oscillators (third row in Fig.(8)) with $b=5 / 2$ and $b=3$, respectively, at least for $N \lesssim 700, N^{1 / 3} \lesssim 8.88$. The agreement extends in particular to the respective occupation numbers, i.e. the degeneracies; of course, the heights of the peaks do not show the same regularity as the corresponding unperturbed problem; nevertheless, even for the heights an overall agreement prevails when comparing with Figs.(8e) and (8f) where the respective unperturbed structures are displayed. For higher values of $N$ we do get deviations which reflect upon the fact that the system is nonintegrable and cannot give complete order in all its results. While the agreement for lower values of $N$ was to be expected from the discussion in the previous section, the extent of the agreement is rather remarkable, especially for $\lambda \approx 0.76 \lambda_{\text {crit }}$ where an astoundingly clean shell structure reoccurs after it partially disappeared for a somewhat smaller value of $\lambda$. It is this recurrence of shell structure which gives rise to the local minima in Fig.(7) as pointed out above.

Of particular interest is Fig.(8b) which refers to the intermediate value $\lambda=0.7 \lambda_{\text {crit }}$ where the genuinely different orbits with winding numbers 5:2 and 3:1 coexist. The long 
wave length fluctuation could well be interpreted as a supershell structure, even though that it is not as clearly pronounced as in a more transparent integrable case [22]. Yet the difference to Fig.(8d) which refers to a larger value of $\lambda$ is striking.

In Figs.(9) we display the square of the modulus of the Fourier transform of the level density, i.e. the function

$$
F(t)=\left|\sum_{n} e^{i \varepsilon_{n} t}\right|^{2} .
$$

The spectrum is taken at $\lambda=0.63 \lambda_{\text {crit }}$ (Fig.(9a)) and at $\lambda=0.76 \lambda_{\text {crit }}$ (Fig(9b)), both spectra refer to $\Lambda=0$ only. The pronounced peaks can be directly associated with the periods of the classical 5:2 and 3:1 orbit, respectively, the periods obtained from Figs.(9) are in perfect agreement with those of the corresponding classical orbits which are found numerically by integrating Eqs.(2). This is a beautiful demonstration of Gutzwiller's trace formula [9]. As expected the frequencies deviate considerably from the unperturbed values, i.e. from the frequency associated with $b=2, \lambda=0$, but also from the frequencies associated with $b=5 / 2$ or $b=3$. In units of the unperturbed value $(b=2)$ we find $T_{5: 2}=1.2$ and $T_{3: 1}=1.4$; the values are larger than unity in accordance with Fig.(2).

Again we stress that the high degree of order which prevails in the superdeformed prolate case when the octupole term is turned on, does not exist in the corresponding oblate, in fact, not even in the spherical case. There, chaotic behaviour becomes manifest for much lesser octupole strength, which results in a complete disappearance of shell structure in the quantum spectrum.

\section{Summary}

The main motivation of our work is the understanding of the nature of shell effects of manybody systems like nuclei or metallic cluster at large quadrupole/octupole deformation. It is the shell structure that is responsible for the existence of superdeformed nuclei which nowadays is a very broad research subject. Metallic clusters provide us with an additional challenge; this topic is attracting more and more researchers who have traditionally been working in nuclear structure. In spite of the different character of the interaction in nuclei (predominantly attractive forces between nucleons) and metallic clusters (repulsive forces between valence electrons), the remarkable similarity in the manifestation of shell structure in experimental data allows analysis of both systems within the same quantum mechanical model. We have investigated the shell structure produced by a quadrupole deformed 
harmonic oscillator with an octupole term. Contrary to the case of spherical potentials our Hamiltonian is nonintegrable. Using the method based on the 'removal of resonances' approach [37], we established a connection between shell structures in the quantum mechanical spectrum and periodic orbits in the corresponding classical problem. In the prolate case the classical problem can effectively be reduced to an integrable set of equations, since the motion in the two coordinates becomes uncoupled. This is in contrast to the spherical and oblate cases, where the motion becomes chaotic when the octupole term is switched on. In the prolate case, at particular values of the strength parameter $\lambda$ the octupole term produces a motion which resembles to a great extent that of a pure but more enhanced prolate oscillator. This provides the classical explanation for the existence of quantum shell structure for prolate/octupole deformed system within the model [11]. At small values of $\lambda$ the most important short orbit is the Lissajou figure with the shape of an 'eight' with winding number 2:1 (top of Fig.(3)).

The fluctuating part of the energy has been extracted from the quantum mechanical single particle spectrum using our procedure which is a variant of the method discussed in [2]. We have found remarkable agreement between manifestations of shell structure for the same values of $\lambda / \lambda_{\text {crit }}$ (Fig.7) and the ones which give rise to stability islands in the Poincaré surfaces of sections relating to the classical short orbits with winding numbers 2:1 $(\lambda \approx 0), 5: 2\left(\lambda / \lambda_{\text {crit }}=0.63\right), 3: 1\left(\lambda / \lambda_{\text {crit }}=0.76\right)$ (Fig. $\left.(4)\right)$. Note that the orbit closest to the triangle orbit, which is one of the most important orbits in a cavity [4, \&, 22], occurs for the quadrupole/octupole deformed harmonic oscillator only at a large value of the octupole strength parameter $\lambda$; the orbit has the winding number 3:2 and is associated with the three islands in the centre of Fig.(4b), but it does not feature in the quantum mechanical spectrum. In the intermediate case $\left(\lambda / \lambda_{\text {crit }}=0.7\right)$ when the orbits with winding numbers 5:2 and 3:1 coexist the long wave length fluctuation could be interpreted as a supershell structure. But due to the narrowness of these orbits the supershell structure is not as clearly pronounced as in a more transparent integrable case 22].

In this paper we placed our emphasis on the superdeformed case which is the most interesting situation when the octupole term is switched on. Shell structure is destroyed for smaller values of the octupole strength for lesser quadrupole deformation or even more so for the spherical case. Our analysis implies that the shell structure favours the superdeformed prolate/octupole in contrast to the oblate/octupole case. The model is only a crude approximation of a realistic situation, and a mean field without consideration of correlations 
(interactions between particles) may not justify a statement as strong as this. Only future experimental data from the newly built facility like EUROGAM and GAMMASPHERE can test our conclusion with regard to nuclei. For clusters one could expect that the process of cluster aggregation and cluster evaporation will follow valleys of the potential energy surface, which is the combined effect of electronic shell structure and ionic lattice. The electronic shell effects should be rather important at relatively small temperature and not for too large number of particles. In the framework of our model the electronic shell structure becomes less pronounced for $N>700$, since higher up in the spectrum the chaotic behaviour may well interfere with the search for shell structure [34]. Alternatively, if shell structure can be observed for larger numbers, our conclusion would be that weaker deformation must prevail. More experimental evidence is needed to assess the situation; such evidence is expected also to shed light on the question of whether clusters provide further insight on the fascinating question of quantum behaviour of classically chaotic systems.

Finally we comment on the $(\vec{l})^{2}$-term in the Nilsson model, which plays a positive role as a phenomenological term in nuclear physics. From our results [34] one should infer that this term destroys shell structure, since it produces chaos in the classical analogous (quadrupole deformed) case, while the experimental data indicate shells for clusters. This is different from nuclear physics where chaos does not show, since one is not interested in too many levels. Further investigations have to provide clarity on this aspect. Work to this effect is in progress.

RGN gratefully acknowledges financial support from DGICYT of Spain. 


\section{A Determination of $\beta$ and $\lambda_{\text {crit }}$}

To study the behaviour of the potential along the line $z=\beta \varrho$ we consider the expression

$$
2 V(\varrho, z) /\left(\varrho^{2} m \omega^{2}\right)=1+\frac{\beta^{2}}{b^{2}}+\lambda \frac{2 \beta^{3}-3 \beta}{\sqrt{1+\beta^{2}}} .
$$

The zero of the derivative gives the maximum/minimum of the leading behaviour of the potential along the line $z=\beta \varrho$. From this we find the relation

$$
\frac{2 \beta}{b^{2}}+\lambda\left(\frac{6 \beta^{2}-3}{\sqrt{1+\beta^{2}}}-\frac{\beta^{2}\left(2 \beta^{2}-3\right)}{\sqrt{1+\beta^{2}}}\right)=0 .
$$

Solving for $\lambda_{\text {crit }}$ we obtain

$$
\lambda_{\text {crit }}=\frac{-2 \beta{\sqrt{1+\beta^{2}}}^{3}}{b^{2}\left(4 \beta^{4}+6 \beta^{2}-3\right)} .
$$

Inserting this for $\lambda$ into the previous equation yields the slope $\beta$ as a function of $b$ when $\lambda=\lambda_{\text {crit }}$, i.e.

$$
\beta=\frac{1}{\sqrt{8}} \sqrt{\frac{9}{2+b^{2}}-6+\frac{\sqrt{3\left(3+2 b^{2}\right)\left(1+14 b^{2}\right)}}{2+b^{2}}} .
$$

\section{B Action and Angle Variables}

The relations between the coordinates and momenta and the actions $J_{i}$ and angles $\alpha_{i}$ are given by

$$
\begin{aligned}
\varrho & =\sqrt{\frac{2 J_{\varrho}}{m \omega}} \sin \alpha_{\varrho} \\
z & =\sqrt{\frac{2 b J_{z}}{m \omega}} \sin \alpha_{z} \\
p_{\varrho} & =\sqrt{2 m \omega J_{\varrho}} \cos \alpha_{\varrho} \\
p_{z} & =\sqrt{\frac{2 m \omega J_{\varrho}}{b}} \cos \alpha_{z} .
\end{aligned}
$$




\section{References}

[1] A. Bohr and B. R. Mottelson, Nuclear Structure (Benjamin, New York, 1975), Vol.2.

[2] V.M.Strutinsky, Nucl.Phys. A122, 1 (1968).

[3] M.Brack, J.Damgaard, A.S.Jensen, H.C.Pauli, V.M.Strutinsky, and C.Y.Wong, Rev.Mod.Phys. 44, 320 (1972).

[4] R.Balian and C. Bloch, Ann.Phys.(N.Y.) 69, 76 (1972).

[5] M.C.Gutzwiller, J.Math.Phys. 12, 343 (1971).

[6] V.M.Strutinsky and A.G.Magner, Fiz.Elem.Chast.At.Yadra 7, 356 (1976) ( Sov.J.Part.Nucl. 7, 138 (1976)).

[7] V.M.Strutinsky, A.G.Magner, S.R.Ofengenden, and T.Dossing, Z.Phys. A283, 269 (1977).

[8] H.Frisk, Nucl.Phys. A511, 309 (1990).

[9] M.C.Gutzwiller Chaos in Classical and Quantum Mechnics Springer, New York Berlin Heidelberg 1990.

[10] R.Arvieu, F.Brut, J.Carbonell, and J.Touchard, Phys.Rev. A35, 2389 (1987).

[11] W.D. Heiss, R.G. Nazmitdinov, and S. Radu, Phys. Rev. Lett. 72, 2351 (1994).

[12] W. A. Heer, Rev. Mod. Phys. 65, 611 (1993).

[13] M. Brack, Rev. Mod. Phys. 65, 677 (1993).

[14] S. Bjornholm, J.Borggreen, O.Echt, K.Hansen, J.Pedersen, and H.D.Rasmussen, Phys. Rev. Lett. 65, 1627 (1990).

[15] C. Brechignas, Ph.Cahuzac, F.Carlier, M.de Frutos, and J.Ph.Roux, Phys.Rev. B47, 2271 (1993).

[16] T. P. Martin, S.Bjornholm, J.Borggreen, C.Brechignas, P.Cahuzac, K.Hansen, and J.Pedersen, Chem. Phys. Lett. 186, 53 (1991). 
[17] K. Clemenger, Phys. Rev. B32, 1359 (1985).

[18] S. Frauendorf and V. V. Pashkevich, Z. Phys. D26, S98 (1993).

[19] C. Yannouleas and U. Landman, Phys. Rev. B48, 8376 (1993).

[20] A. Bulgac and C. Lewenkopf, Phys. Rev. Lett. 71, 4130 (1993).

[21] W. Kohn and L. J. Sham, Phys. Rev. 140, 1133A (1965).

[22] H. Nishioka, K. Hansen, and B. R. Mottelson, Phys. Rev. B42, 9377 (1990).

[23] J. Lerme, Ch.Bordas, M.Pellarin, B.Baguenard, J.L.Vialle, and M.Broyer, Phys. Rev. B48, 9028 (1993).

[24] N. Pavloff and S. C. Creagh, Phys. Rev. B48, 18164 (1993).

[25] W.D.Knight, K.Clemenger, W.A.de Heer, and W.A.Saunders, Phys.Rev. B31, 2539 (1985).

[26] B.Mottelson, Nobel Prize lecture, Rev.Mod.Phys. 48, 375 (1976).

[27] S.Åberg, Nucl.Phys. A557, 17c (1993).

[28] J.L.Egido and L.M.Robledo, Nucl.Phys. A545, 589 (1992).

[29] J.Skalski, P.-H. Heenen, P.Bonche, H.Flocard, and J.Mayer, Nucl.Phys. A551, 109 (1993).

[30] W.Nazarevicz and J.Dobaczewski, Phys.Rev.Lett. 68, 154 (1992).

[31] I.Hamamoto, B.Mottelson, H.Xie, and X.Z.Zhang, Z.Phys. D21, 163 (1991).

[32] J.Blocki, J.-J.Shi, and W.J.Swiatecki, Nucl.Phys. A554, 387 (1993).

[33] P.Rozmej and R.Arvieu, Nucl.Phys. A545, 497c (1992).

[34] W.D. Heiss and R.G. Nazmitdinov, Phys. Rev. Lett., submitted.

[35] V.O.Nesterenko, Fiz.Elem.Chast.At.Yadra 23, 1665 (1992).

[36] M.A.Deleplanque, Nucl.Phys. A557, 39c (1993). 
[37] A.J. Lichtenberg and M.A. Liebermann Regular and Stochastic Motion Springer, New York Berlin Heidelberg 1981.

[38] W.D. Heiss and A.L. Sannino, J. Phys. A: Mathematical and General, 23 (1990) 1167; W.D. Heiss and A.L. Sannino, Phys. Rev. A43 (1991) 4159; W.D. Heiss and A.A. Kotzé, Phys. Rev. A44 (1991) 2403; W.D. Heiss and M. Müller, Phys. Rev. A48, 2558 (1993).

[39] K.Arita, Prog.Theor.Phys. 90, 747 (1993); K.Arita and K.Matsuyanagi, preprint KUNS 1229 (1993).

[40] T.Bengtsson, M.E.Faber, G.Leander, P.Moller, M.Ploszajczak, I.Ragnarsson and S.Åberg, Phys.Scripta 24, 200 (1981). 


\section{Figure captions}

Fig.1 The potential $U(z)$ in units of $m \omega^{2} / 2$ for a few values of $\lambda$ and an intermediate value of $\xi$. Variation of $\xi$ produces basically similar shapes.

Fig. 2 The ratio $\omega_{\varrho} / \omega_{z}$ as a function of $\lambda$ for various values of $\xi$. A genuine $\xi$-dependence is discernible only for $\xi>0.8 \xi_{\text {max }}$.

Fig.3 The major orbits with winding numbers $2: 1(\lambda \gtrsim 0), 5: 2\left(\lambda / \lambda_{\text {crit }}=0.63\right)$ and 3:1 $\left(\lambda / \lambda_{\text {crit }}=0.76\right)$.

Fig.4 Surfaces of sections $(b=2)$ of four orbits for $\lambda=0.63 \lambda_{\text {crit }}$ (top) and of five orbits for $\lambda=0.76 \lambda_{\text {crit }}$ (bottom).

Fig.5 Sections of three orbits for $b=1$ (top) and for $b=1 / 2$ (bottom) for $\lambda=0.5 \lambda_{\text {crit }}$.

Fig.6 Spectrum $(b=2)$ for $\Lambda=0$ (top) and all $\Lambda$ (bottom) as a function of $\lambda$. The energy unit is $\hbar \omega$. Note that there are only avoided level crossings in the top figure which, however, are not resolved.

Fig.7 Contours of $\delta E(N, \lambda)$ with $100<N<700$ as abscissa and $0<\lambda / \lambda_{\text {crit }}<1$ as ordinate. Dark areas represent minima. Volume conservation is taken into account in Figs.(7) and (8).

Fig.8 Shell structure for fixed values of $\lambda$ and $b=2$. The second derivative of $\delta E(N, \lambda)$ is illustrated versus $N^{1 / 3}$ for $\lambda / \lambda_{\text {crit }}=0.15,0.70,0.63$ and 0.76 in (a), (b), (c) and (d), respectively. In (e) and (f) the unperturbed $(\lambda=0)$ structure is displayed for $b=2.5$ and $b=3$, respectively.

Fig.9 Fourier transforms of the modulus square of the level density for $\Lambda=0, b=2$ for $\lambda / \lambda_{\text {crit }}=0.63$ (top) and $\lambda / \lambda_{\text {crit }}=0.76$ (bottom). 